\title{
Características reproductivas de Commelina erecta L. asociadas con su propagación en sistemas agrícolas
}

\author{
Nisensohn, L.A; D.H. Tuesca y J.I. Vitta
}

\begin{abstract}
RESUMEN
Commelina erecta L. se reproduce por rizomas y por dos tipos de semillas. Se estudiaron los requerimientos de luz y temperatura para la germinación, la evolución de la dormición y la viabilidad de las semillas en el suelo, la emergencia de plántulas y macollos y la producción de biomasa aérea y semillas. La germinación de semillas recién cosechadas fue menor a $5 \%$ en las semillas ovoides y en las alargadas fue $91 \%$ con temperaturas alternadas. La longevidad en el suelo varió según el tipo de semilla: a los 12 meses no se recuperaron semillas alargadas mientras que a los 24 meses se recuperó el 14\% de las ovoides. La emergencia de plántulas se inició en octubre y se extendió -de acuerdo al año - hasta diciembre o marzo. La brotación de rizomas abarcó desde octubre a febrero. El total de plántulas emergidas fue independiente de la presencia de plantas provenientes de rizomas y viceversa. En abril se registró un flujo adicional de emergencia de plántulas originadas de semillas producidas durante la misma estación de crecimiento. Las características reproductivas estudiadas permiten explicar la capacidad de esta especie para colonizar y persistir en los sistemas de producción de la región.
\end{abstract}

Palabras clave: Commelina erecta, germinación, emergencia, fecundidad, Iongevidad.

Nisensohn, L.A; D.H. Tuesca and J.I. Vitta, 2011. Reproductive traits of Commelina erecta L. associated with its propagation in agricultural systems. Agriscientia XXVIII: 51-60

\section{SUMMARY}

Commelina erecta reproduces by seeds and rhizomes. Temperature and light requirements for germination, seed dormancy and viability, seedling and tiller dynamics and seed and biomass production were studied. C. erecta shows two types of seeds: ovoid and elongated. After shedding from the parent plant, the percentage of germination of the ovoid seeds was $<5 \%$ whereas of elongated seeds up to $91 \%$ with fluctuating temperatures. Seed longevity varied depending on the type of seed: from 12 months onwards no elongated seeds were recovered, while at 24 months $14 \%$ of the ovoid seeds was recovered. The onset 
of seedling emergence in both years of the experiment was early October and emergence lasted-depending on year- till December or March. The period of rhizome sprout spanned from October to February. Total seedling emergence was independent of the presence of plants originated from rhizomes, and the opposite also holds. In April an additional flux of seedlings originated from seeds produced during the same growing season was recorded. These reproductive traits of $C$. erecta could account for the ecological success of this weed in the agricultural systems.

Key Words: Commelina erecta, germination, emergence, fecundity, Iongevity.

L. A. Nisensohn y D.H. Tuesca. Cátedra de Malezas, Facultad de Ciencias Agrarias de la Universidad Nacional de Rosario. S2125ZAA - CC 14, Zavalla, Santa Fe, Argentina. J.I. Vitta. Fallecido en el año 2006. Correspondencia a L.A. Nisensohn: Inisen@arnet.com.ar

\section{INTRODUCCIÓN}

En la Argentina el modelo productivo de la región pampeana está caracterizado por la preeminencia del cultivo de soja transgénica, la ausencia de laboreo y el uso intensivo de herbicidas, particularmente glifosato (Vitta et al., 1999).

Las medidas de control químico actúan como una poderosa fuerza de selección de la flora de malezas dentro del agroecosistema, generando cambios significativos en la densidad y en la relación de dominancia entre especies (Cousens \& Mortimer, 1995).

Las malezas históricamente han sido consideradas una de las principales limitantes para el rendimiento de los cultivos. Prueba de ello es que en la Argentina la difusión masiva de cultivares de soja resistentes a glifosato (RR) se fundamentó en gran medida en la factibilidad de controlar eficientemente las malezas a un costo relativamente bajo (Vitta et al., 1999).

Del mismo modo que la introducción de la siembra directa generó modificaciones cuali y cuantitativas en las comunidades de malezas (Tuesca et al., 2001; Puricelli y Tuesca, 2005a), la utilización en forma generalizada y continua de glifosato produjo cambios en la flora de malezas (Puricelli \& Tuesca, 2005b; Tuesca \& Puricelli, 2007).

En el marco de la aplicación de este modelo de producción de soja, se han observado incrementos en las poblaciones de Commelina erecta L. (flor de Santa Lucía) (Vitta et al., 2004). Ésta es una especie perenne, herbácea (Faden, 1985; Kissman, 1991; Cabrera y Zardini, 1993; Faden, 1998), que se reproduce a partir de semillas y de rizomas. Sus frutos son cápsulas con dos lóculos dehiscentes y uno indehiscente; en los dehiscentes se producen dos semillas alargadas color negro o marrón y en el indehiscente una semilla ovoide color marrón claro que permanece cubierta por las vestiduras del fruto (Wilson, 1981). En trabajos realizados por Papa et al. (2002) y Nisensohn y Tuesca (2001) se cita a esta especie entre las que presentan tolerancia a glifosato.

Los objetivos de este trabajo fueron: a) determinar los requerimientos de luz y temperatura para la germinación, b) establecer la evolución de la dormición y viabilidad de las semillas en el suelo y c) conocer la dinámica de la emergencia, la producción de biomasa y la fecundidad de plántulas y macollos de Commelina erecta.

\section{MATERIALES Y MÉTODOS}

Los experimentos se realizaron durante los años 2000, 2001 y 2002 en el Campo Experimental de la Facultad de Ciencias Agrarias en la localidad de Zavalla (Lat. $33^{\circ} 01^{\prime}$ S; Long. $60^{\circ} 53^{\prime} \mathrm{O}$ ). Tanto las semillas como los rizomas de la maleza se obtuvieron a partir de plantas aisladas provenientes del mismo sitio experimental.

\section{Requerimientos de temperatura y luz para la germinación}

Los experimentos se llevaron a cabo utilizando cámaras de germinación en las que se establecieron distintos regímenes de temperaturas constantes $\left(20,25,30,35,45^{\circ} \mathrm{C}\right)$ y alternadas $(10 / 20$, $15 / 25,20 / 30,25 / 35,30 / 40,35 / 45^{\circ} \mathrm{C}$ ). Se empleó un fotoperíodo de 10 horas de oscuridad y 14 
horas de luz. La fuente de luz estaba constituida por lámparas fluorescentes que emitían un flujo de fotones de $\sim 40 \mu \mathrm{mol} \mathrm{m} \mathrm{m}^{-2} \mathrm{~s}^{-1}$. Se sembraron 100 semillas en cajas de Petri, con una base de algodón y papel de filtro humedecido con una solución de Benomyl al 2\%. Cada 48 horas se realizó un riego con agua destilada. El diseño experimental fue completamente al azar, con tres repeticiones de cada tipo de semilla (ovoide y alargada). Periódicamente y hasta los 30 días de la siembra, se evaluó el porcentaje de semillas germinadas en todos los tratamientos. El criterio para determinar la germinación fue la visualización de la protusión de la radícula. El requerimiento de luz para la germinación se evaluó comparando dos fotoperíodos: oscuridad (10 h)/luz (14 h) y oscuridad (24 h) a un mismo régimen térmico $\left(25-35^{\circ} \mathrm{C}\right)$. La elección de este régimen se hizo teniendo en cuenta los resultados del experimento realizado con distintos regímenes térmicos y considerando la combinación de temperaturas en la que se registró el máximo porcentaje de germinación.

\section{Cambios en la dormición y viabilidad de las se- millas en el suelo}

Durante el otoño de 2001 -coincidente con el período de dispersión natural de la semilla- se recolectaron semillas y se conservaron en bolsas de papel a temperatura ambiente $\left(20^{\circ} \mathrm{C}\right)$ hasta el momento de ser utilizadas. A mediados de junio del mismo año, se colocaron sobre la superficie del suelo lotes de 100 semillas en bolsas de nylon de 5 por $10 \mathrm{~cm}$, que permitieron el libre intercambio de agua y gases a la vez que impidieron el ingreso de depredadores. El experimento fue completamente aleatorizado con tres repeticiones para cada tipo de semilla. La primera extracción de bolsas con semillas se realizó a los 6 meses de iniciado el experimento y posteriormente cada 3 meses durante 2 años. Las semillas se incubaron en cajas de Petri en condiciones óptimas de temperatura y luz $\left(25-35{ }^{\circ} \mathrm{C} ; 10 \mathrm{~h}\right.$ de oscuridad/ $14 \mathrm{~h}$ de luz). Periódicamente y hasta los 30 días de incubación se cuantificó el número de semillas germinadas. La viabilidad de las semillas remanentes se determinó utilizando el test de tetrazolio (Martin, 1946; Novotna, 1978), considerándose semillas dormidas a aquellas que reaccionaron positivamente a dicho test. El número de semillas recuperadas viables se calculó como la suma de las semillas germinadas en cajas de Petri más las semillas dormidas.

\section{Dinámica de la emergencia, producción de bio- masa y semillas en condiciones de campo}

En un lote libre de $C$. erecta destinado durante los últimos diez años al monocultivo de soja en siembra directa se realizaron dos ensayos, el primero de ellos se instaló en 2000 y el segundo en 2001. El suelo fue caracterizado como argiudol vértico $(70 \%$ de limo, $25 \%$ de arcilla y $5 \%$ de arena), con una pendiente de 0 a $2 \%$, un contenido de materia orgánica de 3\% y un $\mathrm{pH}$ de5,8.

En la primera quincena de octubre de ambos años, se delimitaron parcelas de 1,5 por 0,7 m. En ellas se sembraron superficialmente 200 semillas (la mitad de cada tipo) y las plantas originadas de rizomas se obtuvieron sembrando cuatro trozos de rizomas con dos nudos cada uno (cada nudo posee tres a cuatro yemas), a fin de obtener efectivamente cuatro plantas $/ \mathrm{m}^{2}$. Así quedaron definidos tres tratamientos: 1) rizomas, 2) semillas + rizomas 3) semillas. Las semillas se sembraron al azar y los trozos de rizomas se marcaron con fichas para diferenciar luego las plantas emergidas.

El diseño experimental fue en bloques completos al azar con seis repeticiones. El cultivo de soja (cv. Asgrow RR 640/600) se sembró el 11/11/2000 y el 12/12/2001 a una distancia de 0,7 m entre hileras, y no se realizó ningún tratamiento de fertilización. Antes de la siembra de $C$. erecta, las malezas se controlaron con glifosato (960 g i.a. ha-1). Posteriormente, las malezas gramíneas fueron controladas con haloxyfop (360 g i.a. ha-1) y las latifoliadas fueron eliminadas manualmente.

El patrón de emergencia de ambas formas de propagación se obtuvo registrando semanalmente -mediante anillos de diferentes colores- la emergencia de plántulas originadas de ambos tipos de semillas (sin diferenciarlas) y de los macollos (provenientes de las yemas de los rizomas). Las plántulas y macollos emergidos durante una semana constituyeron una cohorte. Se consideraron plántulas los individuos provenientes de semillas con dos a tres hojas y macollos a los vástagos basales con dos a tres hojas que provenían de rizomas.

En ambos años, durante el otoño se registró un flujo adicional de emergencia de plántulas en todos los tratamientos, el cual fue analizado en forma separada al flujo ocurrido en la primavera y verano.

Durante 2001 se estimó el número de semillas producidas por parcela a partir del recuento de las inflorescencias. Se consideró que en cada inflorescencia se formaban cuatro frutos con tres semillas cada uno. En todos los casos se discriminó el aporte de las plantas provenientes de rizomas y de semillas. Al finalizar el ciclo, la biomasa aérea de las plantas fue cosechada en forma individual 
y se secó en estufa a $80^{\circ} \mathrm{C}$ durante 72 horas para determinar su peso seco.

Las precipitaciones y las temperaturas medias mensuales se obtuvieron a partir de la información suministrada por la estación meteorológica del Campo Experimental de la Facultad de Ciencias Agrarias de la Universidad Nacional de Rosario. En el año 2000, durante el desarrollo del ensayo, las Iluvias fueron de 1009,3 mm, mientras que en 2001 fueron de 765,5 mm; la media histórica es de $676 \mathrm{~mm}$. El rango de temperaturas medias durante el mismo período varío entre $17,4^{\circ} \mathrm{C}$ y $24,5^{\circ} \mathrm{C}$ en el año 2000 y entre $17,8^{\circ} \mathrm{C}$ y $24^{\circ} \mathrm{C}$ en 2001 , mientras que las correspondientes medias históricas varían entre $17,1^{\circ} \mathrm{C}$ y $23,7^{\circ} \mathrm{C}$.

\section{Análisis estadístico}

El porcentaje de germinación en los distintos regímenes térmicos evaluados se comparó mediante un análisis de la variancia, y las diferencias entre las medias se analizaron mediante una prueba de $\operatorname{LSD}(p=0,05)$. Las diferencias entre los tratamientos con y sin luz se analizaron mediante una prueba de $t(p=0,05)$.

En cada fecha de extracción y para cada tipo de semilla se analizaron las diferencias entre el porcentaje de las semillas recuperadas, dormidas y viables en la superficie del suelo mediante una prueba de $t(p=0,05)$.

Las diferencias en el número total de plántulas y de macollos emergidos durante la primavera y el inicio del verano, así como entre el número de plántulas emergidas en el otoño en los distintos tratamientos, se evaluaron mediante un análisis de la variancia con dos factores (año y tratamientos), considerando la interacción entre ambos. Las diferencias entre medias fueron comparadas con la prueba de comparaciones múltiples LSD $(p=0,05)$.

La producción total de biomasa y de semillas en los diferentes tratamientos se evaluó mediante un análisis de la variancia, mientras que las diferencias de medias fueron analizadas mediante una prueba de LSD $(p=0,05)$.

La relación entre el número de semillas producidas y el peso seco aéreo final de las plantas se estimó utilizando regresiones lineales.

En todos los casos que correspondió, se realizaron transformaciones en las variables analizadas de manera de homogeneizar la variancia y luego se volvieron a transformar para su presentación; en el caso de valores expresados como porcentajes se empleó el arco seno de la raíz cuadrada de $(x+0,5)$, y en el caso de densidad se empleó raíz cuadrada de $x$.

\section{RESULTADOS}

\section{Requerimientos de temperatura y luz para la germinación}

El porcentaje de germinación de las semillas ovoides en todos los casos fue bajo ( $<5 \%)$. En el caso de las semillas alargadas, los valores más altos de germinación $(81,7$ a 91,0\%) se registraron con alternancias de $15 / 25,20 / 30$ y $25 / 35^{\circ} \mathrm{C}$ (Tabla 1). Con temperaturas constantes, la máxima germinación $(68,3 \%)$ se obtuvo a $30^{\circ} \mathrm{C}$. La germinación en condiciones de alternancia de luz y oscuridad no difirió estadísticamente de la obtenida en oscuridad permanente, y alcanzó valores de 95,8 $( \pm 1,4) \%$ y $89,2( \pm 5,8) \%$, respectivamente.

Tabla 1: Porcentaje de germinación de las semillas alargadas de $C$. erecta expuestas a diferentes regímenes de temperatura. Entre paréntesis se indican los desvíos estándar.

\begin{tabular}{|c|c|c|}
\hline $\begin{array}{c}\text { Temperatura } \\
\left({ }^{\circ} \mathrm{C}\right)\end{array}$ & \multicolumn{2}{|c|}{$\begin{array}{c}\text { Germinación } \\
(\%)\end{array}$} \\
\hline 20 & $5,0(0,00)$ & $d$ \\
\hline 25 & $25,0(10,00)$ & c \\
\hline 30 & $68,3(5,77)$ & $b$ \\
\hline 35 & $23,3(2,88)$ & c \\
\hline 45 & $0,0(0,00)$ & e \\
\hline $10-20$ & $35,0(13,22)$ & c \\
\hline $15-25$ & $81,7(12,58)$ & $a b$ \\
\hline $20-30$ & $90,7(2,08)$ & a \\
\hline $25-35$ & $91,0(1,73)$ & a \\
\hline $30-40$ & $5,0(8,66)$ & de \\
\hline $35-45$ & $1,7(2,88)$ & de \\
\hline
\end{tabular}

Letras distintas indican diferencias significativas $(p=0,05)$ según la prueba de LSD

\section{Cambios en la dormición y viabilidad de las se- millas en el suelo}

Al inicio del experimento, la viabilidad de ambos tipos de semillas fue $96 \%$ y $98 \%$ en las semillas ovoides y alargadas respectivamente. En ese momento, los porcentajes de dormición difirieron marcadamente de acuerdo al tipo de semilla: 33\% para las semillas alargadas y $96 \%$ para las ovoides (Tabla 2). El comportamiento de las semillas en el suelo varió sustancialmente de acuerdo al tipo de semilla considerado; para cada fecha de extracción se observaron diferencias significativas entre las semillas de ambos tipos recuperadas, dormidas y viables a lo largo de todo el experimento. A los 6 meses, sólo se recuperó el 12\% de las semillas alargadas, las cuales en su gran mayoría se encontraban viables y despiertas. Por el contrario, en esa misma fecha se recuperó el $75 \%$ de las semillas ovoides, todas ellas viables y dormidas. A 
Tabla 2: Cambios en la fracción de semillas recuperadas, dormidas y viables de ambos tipos (alargadas y ovoides) de $C$. erecta durante su permanencia en la superficie del suelo. Entre paréntesis se indican los desvíos estándar.

\begin{tabular}{|c|c|c|c|c|c|c|}
\hline \multirow[t]{2}{*}{ Meses } & \multicolumn{2}{|c|}{ Semillas recuperadas (\%) } & \multicolumn{2}{|c|}{ Semillas dormidas (\%) } & \multicolumn{2}{|c|}{ Semillas viables (\%) } \\
\hline & Alargadas & Ovoides & Alargadas & Ovoides & Alargadas & Ovoides \\
\hline 0 & & & $33(1,15)$ & $96(1)$ & $98(1)$ & $96(1)$ \\
\hline 6 & $12(1)$ & $75,3(2,08)$ & $1(0)$ & $74,3(1,15)$ & $11(1)$ & $74,3(1,15)$ \\
\hline 9 & $10(1)$ & $63(1)$ & $1,3(0,57)$ & $36,6(1,52)$ & $8,7(0,58)$ & $59(2,64)$ \\
\hline 12 & $7,7(2,5)$ & $50(5,29)$ & $1,7(0,57)$ & $37,3(7,63)$ & $6,7(2,51)$ & $47,3(2,88)$ \\
\hline 15 & 0 & $46,7(1,52)$ & 0 & $22,6(2,31)$ & 0 & $35(1)$ \\
\hline 18 & 0 & $41,7(7,09)$ & 0 & $21(1,73)$ & 0 & $29,6(4,51)$ \\
\hline 21 & 0 & $28,7(5,51)$ & 0 & $17(2,64)$ & 0 & $21,3(4,16)$ \\
\hline 24 & 0 & $13,7(0,58)$ & 0 & $9(1)$ & 0 & $9(1)$ \\
\hline
\end{tabular}

*Para cada fecha se observaron diferencias significativas entre las semillas de ambos tipos (alargadas y ovoides) recuperadas, dormidas y viables a lo largo de todo el experimento según la prueba de $t(p=0,05)$.

partir de los 12 meses no se recuperaron semillas alargadas mientras que a los 24 meses aún se recuperó el $13,7 \%$ de las semillas ovoides. De ese porcentaje, $66 \%$ correspondió a semillas viables y dormidas.

\section{Dinámica de la emergencia, producción de bio- masa y semillas en condiciones de campo}

En ambos años la emergencia de la primera cohorte de plántulas se inició 20 días después del comienzo del experimento, es decir a fines del mes de octubre. En 2000 el período de emergencia abarcó hasta los primeros días de diciembre, mientras que en 2001 se prolongó hasta inicios de marzo. La floración de las plantas provenientes de semillas se inició en la primera quincena de diciembre y se extendió hasta comienzos de abril (Figura 1).

La emergencia de la primera cohorte de macollos se inició a mediados de octubre, aproximadamente 10 días después del comienzo del experi-

Tabla 3: Densidad $\left(n^{\circ} / \mathrm{m}^{2}\right)$ de a) plántulas y b) macollos de $C$. erecta en los distintos tratamientos: (1) rizomas; (2) rizomas + semillas; (3) semillas. Entre paréntesis se indican los desvíos estándar.

a)

\begin{tabular}{|c|c|c|c|}
\hline & Tratamientos & \multicolumn{2}{|c|}{ Años } \\
\hline & & 2000 & 2001 \\
\hline \multirow{3}{*}{ Macollos } & 1 & $159,8(17,21)$ & $191,0(30,82)$ \\
\hline & 2 & $145,4(18,41)$ & $179,0(19,83)$ \\
\hline & Promedio & $152,5(18.71) \mathrm{a}$ & $184.9(25.62) b$ \\
\hline \multicolumn{4}{|c|}{ 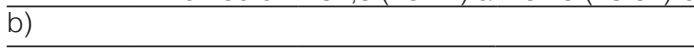 } \\
\hline & \multirow[t]{2}{*}{ Tratamientos } & \multicolumn{2}{|c|}{ Años } \\
\hline & & 2000 & 2001 \\
\hline \multirow{3}{*}{ Plántulas } & 2 & $50,0(8,01)$ & $77,4(23,21)$ \\
\hline & 3 & $40,0(9,81)$ & $76,0(12,81)$ \\
\hline & Promedio & $45,0(10,0) a$ & $75.7(17.84) b$ \\
\hline
\end{tabular}

Letras distintas indican diferencias significativas entre años según una prueba de LSD $(p=0,05)$ mento. La mayor emergencia se produjo a fines de octubre, extendiéndose hasta los primeros días de febrero. La floración de las plantas provenientes de rizomas se inició en la primera quincena de noviembre y se extendió hasta comienzos de abril (Figura 2).

No se observó interacción entre años y tratamientos en el número total de plántulas y de macollos emergidos durante la primavera y el inicio del verano. La emergencia, tanto de macollos como de plántulas, difirió significativamente entre años y fue mayor en 2001. En ambos años, no hubo diferencias significativas entre el número total de plántulas emergidas en el tratamiento 2 (semillas + rizomas) y el tratamiento 3 (semillas), por lo tanto la presencia de macollos no afectó la emergencia de plántulas. Asimismo, el número total de macollos emergidos en el tratamiento 1 (rizomas) no difirió del tratamiento 2 (semillas+ rizomas), es decir que la emergencia de macollos fue independiente de la presencia de plántulas en las parcelas (Tabla 3).

Durante el mes de abril se registró un nuevo flujo de emergencia de plántulas en todos los tratamientos. No se observó interacción entre años y tratamientos; el número de plántulas emergidas en cada tratamiento fue similar en ambos años y fue significativamente mayor en los tratamientos en los que había plantas provenientes de rizomas (Tabla 4).

La relación entre la biomasa aérea y la fecundidad fue diferente de acuerdo al origen de las plantas (Figura 3). La pendiente de la regresión entre ambas variables fue mayor cuando las plantas provinieron de semillas - de menor peso- que cuando provinieron de rizomas - de mayor peso-, lo que indica que en el primer caso existió una mayor partición de recursos a la producción de semillas por unidad de biomasa aérea.

La producción por unidad de área, tanto de biomasa aérea como de semillas, no difirió significati- 


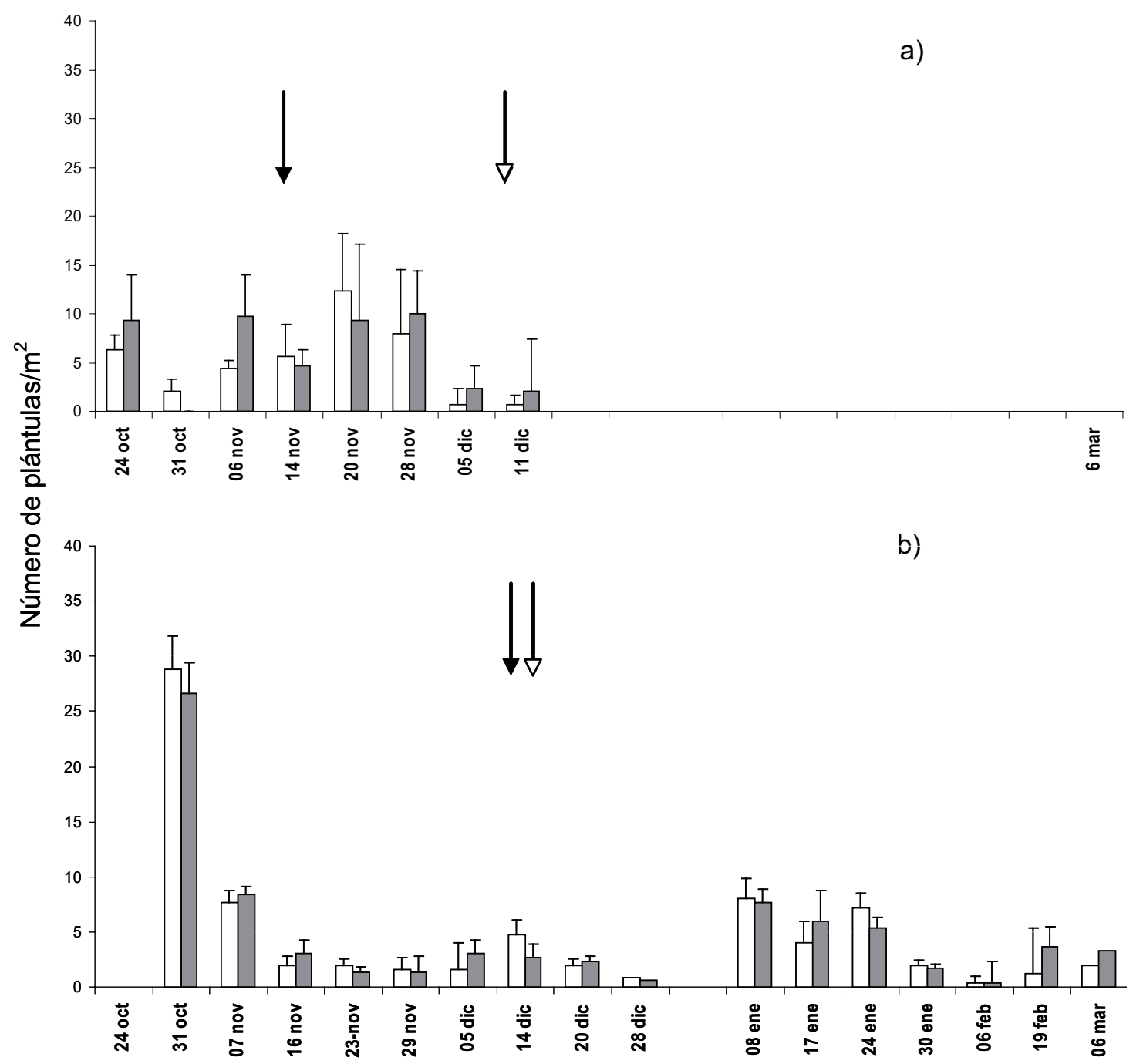

Figura 1: Emergencia (plántulas $/ \mathrm{m}^{2}$ ) de las distintas cohortes de $C$. erecta. a) 2000 ; b) 2001; $(\square)$ sin plantas originadas de rizomas y ( $\square$ ) con plantas originadas de rizomas; ( \indica la siembra del cultivo y $\downarrow$

Tabla 4: Densidad ( $\mathrm{n} / \mathrm{m} 2)$ de plántulas de C. erecta emergidas durante el mes de abril en los distintos tratamientos. (1) rizomas; (2) rizomas + semillas; (3) semillas. Entre paréntesis se indican los desvíos estándar.

\begin{tabular}{ccccc}
\hline & \multicolumn{3}{c}{ Años } \\
\hline & \multicolumn{5}{c}{ Tratamientos } & 2000 & 2001 & Promedio \\
\hline \multirow{3}{*}{ Plántulas } & 1 & $76,7(15,27)$ & $72,0(8,71)$ & $74,3(11.41) \mathrm{a}$ \\
& 2 & $85,3(15,01)$ & $80,0(8,71)$ & $82.7(11.36) \mathrm{a}$ \\
& 3 & $30,7(7,02)$ & $43,3(19,08)$ & $37,0(14.57) \mathrm{b}$ \\
\hline
\end{tabular}

Letras distintas indican diferencias significativas entre tratamientos según una prueba de LSD $(p=0,05)$ 

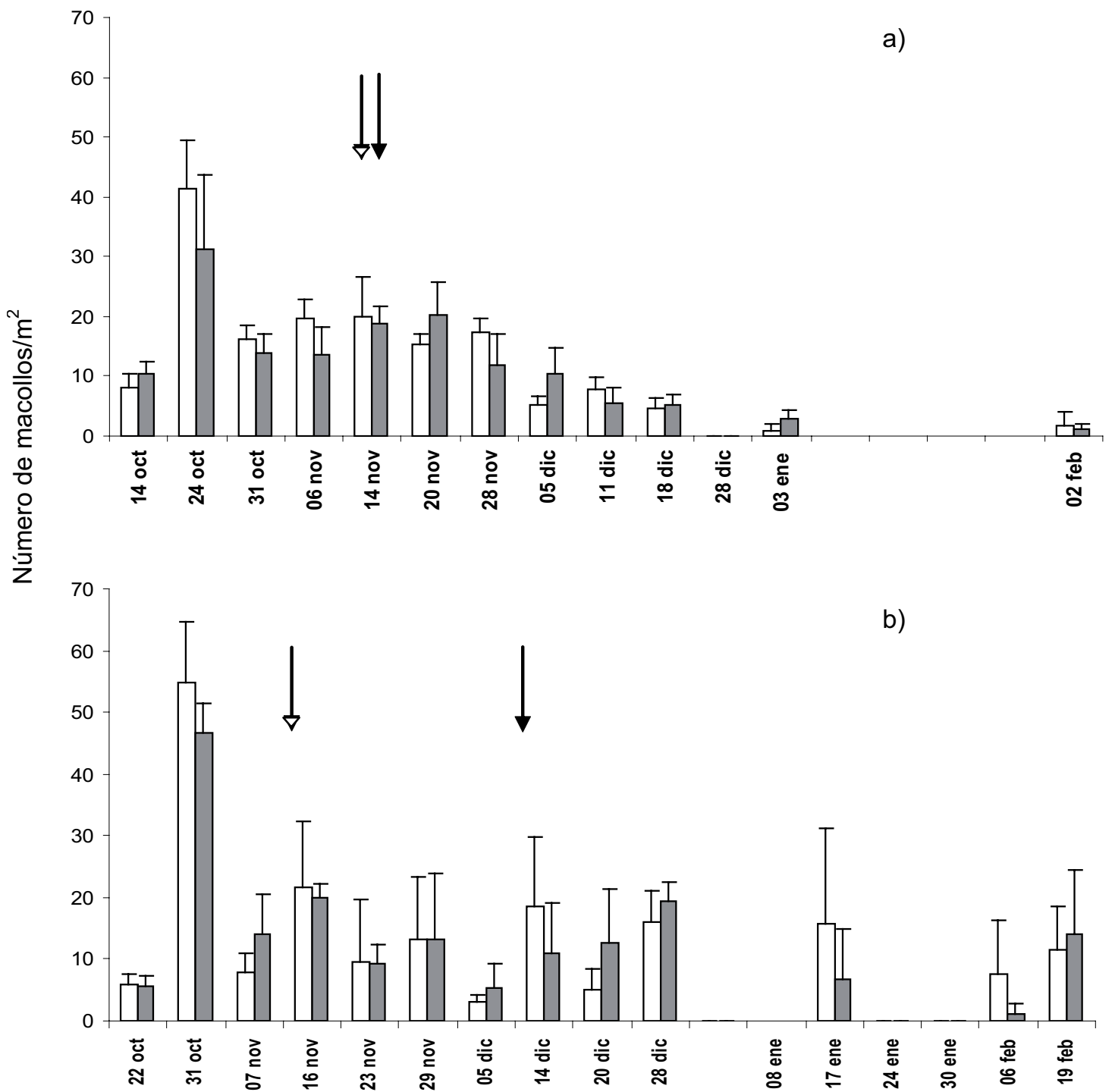

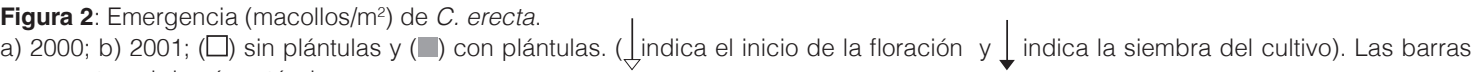
representan el desvío estándar.

vamente entre tratamientos (Figuras 4 y 5 ).

\section{DISCUSIÓN}

\section{Requerimientos de temperatura y luz para la germinación}

Las temperaturas alternas promovieron la germinación de semillas alargadas de $C$. erecta recién dispersadas, la que alcanzó los porcentajes máximos en un rango relativamente amplio de regimenes (desde $15 / 25$ hasta $25 / 35^{\circ} \mathrm{C}$ ). No obstante ello, una fracción de la población de semillas germinó también a temperaturas constantes (hasta $68 \%$ a $30{ }^{\circ} \mathrm{C}$ ), lo cual sugiere una variabilidad importante de la población de la maleza en sus requerimientos para germinar. Hiratsuka (1991), trabajando con semillas de Commelina communis, encontró una respuesta similar, ya que la germinación a temperaturas constantes no superó el $60 \%$ mientras que fue mayor a $80 \%$ con alternancias de $10 / 30$ y $10 / 20^{\circ} \mathrm{C}$.

La germinación de $C$. erecta fue insensible a la luz. La información acerca de la importancia de este factor en especies emparentadas con $C$. erecta es escasa y en ciertos casos contradictoria. 


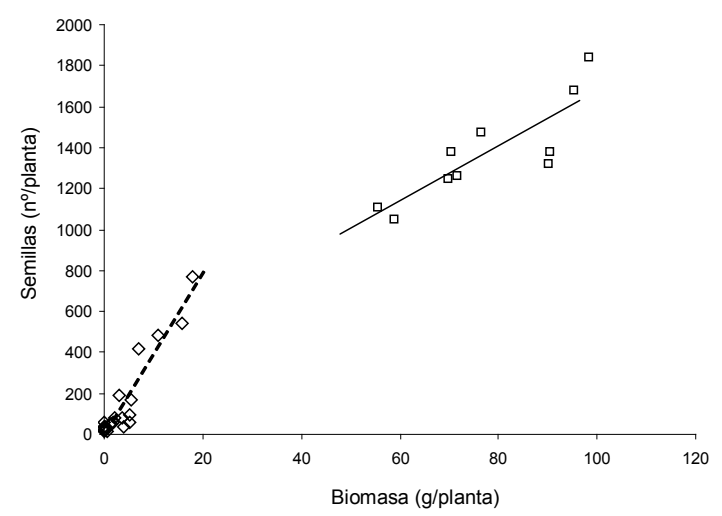

Figura 3: Relación entre la fecundidad y la biomasa aérea de plantas de $C$. erecta al finalizar el ciclo.

$(\diamond)$ Plantas provenientes de semillas; $y=38,241 x+3,8473 ; r^{2}$ $=0,8941$

( $\square$ ) Plantas provenientes de rizomas; $y=13,641 x+311,54 ; r^{2}=$ 0,7191

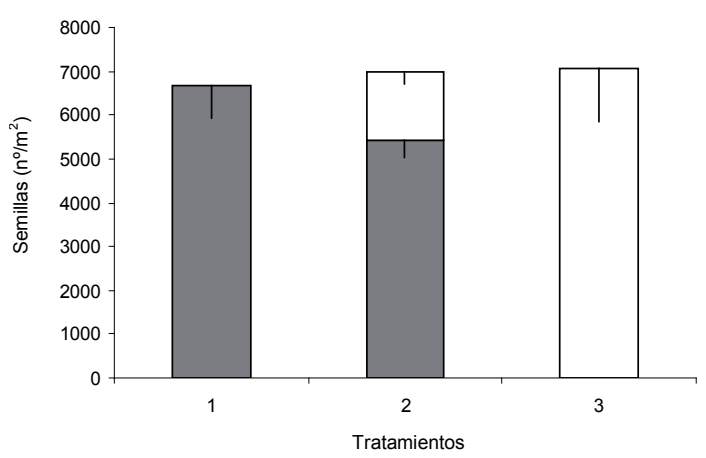

Figura 5: Producción de semillas de $C$. erecta en los diferentes tratamientos. (1) rizomas; (2) rizomas + semillas y (3) semillas.

$(\square)$ aporte de los individuos originados de rizomas y ( $\square$ ) aporte de los individuos originados de semillas. Las barras representan el desvío estándar.

En Commelina benghalensis, por ejemplo, algunos estudios demuestran una respuesta positiva a la luz (Walker \& Evenson, 1985), mientras que otros sostienen que la respuesta es neutra (Gonzalez \& Haddad, 1995). Matsuo et al. (2004) observaron que, en las semillas aéreas de $C$. benghalensis, la luz promovió la germinación sólo de las semillas más pequeñas.

\section{Cambios en la dormición y viabilidad de las se- millas en el suelo}

Los dos tipos de semillas de $C$. erecta tuvieron un comportamiento diferencial, tanto en cuanto a su nivel de dormición inicial como en su longevidad en el suelo. El $66 \%$ de las semillas alargadas se encontraban despiertas al momento de desprenderse de la planta madre. Una vez en la superficie

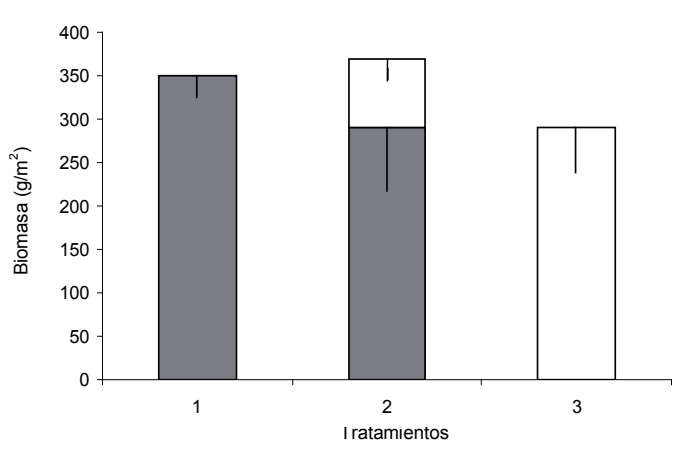

Figura 4: Producción de biomasa aérea de $C$. erecta al finalizar el ciclo, en los diferentes tratamientos. (1) rizomas; (2) rizomas + semillas y (3) semillas.

$(\square)$ aporte de individuos originados de rizomas y $(\square)$ aporte de los individuos originados de semillas. Las barras representan el desvío estándar.

del suelo, la totalidad de esas semillas perdieron la viabilidad en el término de un año. Por el contrario, las semillas ovoides presentaron una elevada dormición inicial y mayor longevidad que las semillas alargadas: luego de dos años en el suelo aún permanecía viable $14 \%$ de la población original. Teniendo en cuenta que las bolsas en que se encontraban las semillas impedían el ingreso de depredadores, es factible inferir que las semillas que no se recuperaron en las sucesivas exhumaciones germinaron previamente como consecuencia de la pérdida de la dormición. Este polimorfismo somático observado en $C$. erecta también ocurre en otras especies del mismo género. Commelina diffusa presenta tres tipos de semillas que difieren tanto en su dormición como en el porcentaje y velocidad de germinación (Santos et al., 2004). A su vez, C. benghalensis posee cuatro tipos de semillas, cada una de ellas con un comportamiento germinativo particular derivado de diferencias en la cubierta seminal (Budd et al., 1979; Walter \& Evenson, 1985; Kim et al., 1990; Rodrigues \& Pitelli, 1994. En el caso de $C$. erecta, los dos tipos de semillas le permiten ampliar el rango de condiciones para su regeneración, lo que favorece la adaptación de la maleza a ambientes variables como los agroecosistemas (Harper et al., 1970; Venable \& Brown, 1988).

\section{Dinámica de la emergencia, producción de bio- masa y semillas en condiciones de campo}

El período de emergencia de macollos y plántulas fue mucho más extendido en 2001 que en 2000. Esa emergencia más prolongada se tradujo a su vez en un mayor número final de plántulas y macollos. En el género Commelina la humedad 
es un factor sumamente determinante durante la emergencia. Por ejemplo, en su hábitat nativo, $C$. benghalensis es una maleza de la estación Iluviosa, y su establecimiento está estrechamente asociado al nivel de humedad edáfica (Holm et al., 1977; Kaul et al., 2002,). En Japón, la emergencia de $C$. benghalensis en campos irrigados se extiende dos meses más que en los no irrigados (Matsuo et al., 2004). Sin embargo, en el caso de los experimentos aquí analizados, la humedad no pareció limitar la emergencia ya que las precipitaciones durante el período de emergencia en 2000 fueron superiores a las de 2001 (1009,3 mm y $765,5 \mathrm{~mm}$ respectivamente). La fecha de siembra de soja pudo haber afectado el patrón y el número de individuos emergidos. En 2000 la soja se sembró un mes antes que en 2001, de manera que en este último año el cierre del canopeo del cultivo se postergó en el tiempo. Dicho canopeo puede modificar el ambiente térmico y lumínico detectado por la maleza; en particular, reduce la alternancia térmica y aumenta la proporción de radiación rojo lejano en la superficie del suelo. Como ya fue mencionado, una fracción importante de las semillas de $C$. erecta responde positivamente a la alternancia de temperatura, de manera que es esperable que el anticipo de la siembra y la consecuente cobertura temprana del cultivo reduzca la emergencia de plántulas. En el caso de los individuos provenientes de rizomas, es posible que el cierre del canopeo provoque cambios en la calidad de la luz que controlen la emergencia de macollos (Martinez-Ghersa et al., 2000).

Durante el mes de abril se observó la emergencia de un nuevo flujo de plántulas en todos los tratamientos (Tabla 4). El hecho de que se registrara emergencia de plántulas en el tratamiento donde sólo se plantaron rizomas permite suponer que esas plántulas provinieron de semillas producidas durante la misma la estación de crecimiento. El número de plántulas fue mayor en los tratamientos que incluían originalmente rizomas. Este hecho puede atribuirse a que las plantas provenientes de rizomas florecieron un mes antes que las originadas de semillas y por lo tanto habrían anticipado su aporte de semillas al suelo. Teniendo en cuenta el polimorfismo somático de $C$. erecta antes discutido, es posible que las plántulas emergidas durante abril provengan de semillas alargadas, caracterizadas por una menor dormición al momento de ser liberadas de la planta madre.

La producción por unidad de área de biomasa aérea y de semillas no difirió entre tratamientos. Esto sugiere que cuando se mezclaron ambas formas de propagación, se manifestó competencia entre ellas. En esa situación, las plantas provenientes de semillas redujeron en mayor proporción tanto su biomasa como su fecundidad, de lo cual se deduce que su habilidad competitiva fue menor que la de las plantas provenientes de rizomas (Nisensohn, 2006).

El análisis de la pendiente de la regresión entre la fecundidad de plantas y su biomasa indica que los individuos provenientes de semillas asignaron proporcionalmente más recursos a la reproducción que los originados de rizomas. Harper (1967) sostiene que el esfuerzo reproductivo de una maleza está inversamente relacionado con el grado de madurez del hábitat que explora. En el caso de $C$. erecta, la estrategia colonizadora (semillas) particionó más a semillas que la estrategia asociada con hábitats más maduros (rizomas). Otra explicación es que la diferente partición de recursos puede estar relacionada con las variaciones en el peso de las plantas de acuerdo a su origen, el cual fue mayor en las provenientes de rizomas. En ese sentido, estudios en Solidago sp. demostraron que el esfuerzo reproductivo está inversamente relacionado con el tamaño de las plantas (Abrahamson \& Gadgil, 1973).

\section{CONCLUSIONES}

La capacidad de $C$. erecta para reproducirse de forma sexual y asexual, la presencia de dos tipos de semillas con alta viabilidad y diferentes grados de dormición, su prolongado período de emergencia de plántulas, de floración y producción de semillas y su baja sensibilidad al glifosato, permiten explicar la capacidad de esta especie para colonizar y persistir en los sistemas de producción de la región.

\section{BIBLIOGRAFÍA}

Abrahamson, W.G. and M. Gadgil, 1973. Growth form and reproductive effort in goldenrods (Solidago, Compositae), American Naturalist 107:651-661.

Budd, G.; P. Thomas and J. Allison, 1979. Vegetative regeneration, depth of germination and seed dormancy in Commelina benghalensis L. Research notes, Rhodesian Journal of Agricultural Research 17:151-153.

Cabrera, A.L. y E.M. Zardini, 1993. Manual de la Flora de los alrededores de Buenos Aires. $2^{\circ}$ Edición. Editorial ACME, Buenos Aires, 755 pp.

Cousens, R. and M. Mortimer, 1995. Dynamics of Weed Populations. Cambridge University Press, Cambrige, U.K. 332 pp.

Faden, R., 1985. Commelinaceae, in The families of the monocotyledons. Eds. R. M. T. Dahlgren, H. T. Clifford, and P. F. Yeo. Berlin: Springer Verlag, pp. 381-387. 
Faden, R.B., 1998. Commelinaceae, in: Kubitzki, K. (Ed.), The families and genera of vascular plants, Vol. 4. Flowering plants, monocotyledons, Alismatanae and Commelinanae (except Gramineae). Berlin and Heidelberg. Springer, pp.109-128.

Gonzalez, C.B. and C.R.B. Haddad, 1995. Efeito da luz e temperature na floração de sementes de Commelina benghalensis L. Arquivos de Biologia e Tecnologia 38 (2): 651-659.

Harper, J.L., 1967. A Darwinian approach to plant ecology. Journal of Ecology 55:247-270.

Harper, J.L.; P.H. Lovell and K.G.Moore, 1970. The shapes and sizes of seeds. Annual Review of Ecology and Systematics 1: 327-356.

Hiratsuka, A., 1991. The effect of temperature fluctuation on germination of Commelina communis. Ecological Review 22 (2): 93-97.

Holm, I.G., D.L. Plucknett, J.V. Pancho and J.P. Herberger, 1977. The World's Worst Weeds. Distribution and biology. East-west Center, University Press of Hawaii, Honolulu. 609 pp.

Kaul, V.; N. Sharma and A.K. Koul, 2002. Reproductive effort and sex allocation strategy in Commelina benghalensis L., a common monsoon weed. Botanical Journal of the Linnean Society 140:403-413.

Kim, S.Y.; S.K. De Datta and B.L. Mercado, 1990. The effect of chemical and heat treatments on germination of Commelina benghalensis L. aerial seeds. Weed Research 30: 109-116.

Kissmann, K. G., 1991. Plantas Infestantes e Nocivas. Tomo I, Sao Bernardo do Campo. BASF Brasileira S.A. $825 \mathrm{pp}$.

Martin, A. C., 1946. The comparative internal morphology of seeds. The American Midland Naturalist 36: 513660.

Martinez-Ghersa, M.A.; C.M. Ghersa and E.H. Satorre, 2000. Coevolution of agricultural systems and their weed companions: implications for research. Field Crops Research 67:181-190.

Matsuo, M.; H. Michinaga, H. Terao and E. Tsuzuki, 2004. Aerial seed germination and morphological characteristics of juvenile seedlings in Commelina benghalensis L. Weed Biology Management 4:148-153.

Nisensohn, L. y D. Tuesca, 2001 "Especies de Malezas asociadas al nuevo modelo productivo de la región: Commelina erecta". Revista Agromensajes. Fac Cs. Agrarias, UNR, 5:10-11.

Nisensohn, L. A., 2006. Tesis: "Caracterización de la estrategias de propagación de Commelina erecta en sistemas agrícolas". Maestría en Manejo y Conservación de Recursos Naturales. Escuela de Graduados.
Facultad de Ciencias Agrarias. UNR. 92 pp.

Novotna, E., 1978. Die prapartion der Blumensamenbryonen fur die anwendony des Tetrazolium tests. Seed Science and Technology 6: 809-868.

Papa, J.C; E. Puricelli y J.C. Felizia, 2002. Malezas Tolerantes a Herbicidas en Soja. Revista IDIA XXI (3): 64-67.

Puricelli, E. y D. Tuesca, 2005a. Efecto del sistema de labranza sobre la dinámica de la comunidad de malezas en trigo y barbechos de secuencias de cultivos resistentes a glifosato. Agriscientia 22, 69-78.

Puricelli, E and D. Tuesca, 2005b. Weed density and diversity under glyphosate-resistant crop sequences. Crop Protection 24: 533-542.

Rodrigues, B and R.A. Pitelli, 1994. Quebra de dormencia em sementes de Commelina benghalensis. Planta Daninha 12 (2): 106-110.

Santos, I.C.; L.D. Tuffi Santos, F.A. Ferreira and G.V. Miranda, 2004. Germinação de Sementes de Commelina diffusa cultivada em casa de Vegetação. Anais XXIV Congresso Brasileiro da Ciencia das Plantas Daninhas. P. 31-32. 24 al 28 de mayo de 2004. San Pablo. Brasil.

Tuesca, D.; E. Puricelli and J.C. Papa, 2001. A long-term study of weed flora shifts in different tillage systems. Weed Research 41: 369-382.

Tuesca, D. and E. Puricelli, 2007. Effect of tillage systems and herbicide treatments on weed abundance and diversity in a glyphosate resistant crop rotation. Crop Protection 26: 1765-1770.

Venable, L.D. and J.S. Brown, 1988. The selective interactions of dispersal, dormancy, and seed size as adaptations for reducing risk in variable environments. The American Naturalist 131(3): 360-384.

Vitta, J.; D. Faccini, L. Nisensohn, E. Puricelli, D. Tuesca y E. Leguizamón, 1999. Las malezas en la región sojera núcleo Argentina: situación actual y perspectivas. 47 pp.

Vitta, J.I.; D. Tuesca and E. Puricelli, 2004. Widespread use of glyphosate tolerant soybean and weed community richness in Argentina. Agriculture, Ecosystems and Environment 103: 621-624.

Walker, S. R. and J.P. Evenson, 1985. Biology of Commelina benghalensis L. in south-eastern Queensland. 2. Seed dormancy, germination and emergence. Weed Research 25: 245-250.

Wilson, A.K., 1981. Commelinaceae-A Review of the Distribution, Biology and Control of the Important Weeds Belonging to this family. Tropical Pest Management 27(3): 405-418. 